\title{
Students' Mental Prototypes for Functions and Graphs
}

\author{
David Tall \& MdNor Bakar \\ Mathematics Education Research Centre \\ University of Warwick \\ COVENTRY CV4 7AL \\ U.K.
}

\begin{abstract}
This research study investigates the concept of function developed by English Alevel students. The hypothesis is that students develop prototypes for the function concept in much the same way as they develop prototypes for concepts in everyday life. The definition of the function concept, though given in the curriculum, is not stressed and proves to be inoperative, with student understanding of the concept reliant on properties of familiar prototype examples. Investigations reveal significant misconceptions. For example, threequarters of a sample of students starting a university mathematics course considered a constant function is not a function in at least one of its graphical or algebraic forms, and threequarters thought that a circle is.
\end{abstract}

The concept of a function begins in the U.K. National Curriculum at around the age of nine (algebra attainment target 6, level 3, DES 1989), yet the definition of a function causes great difficulties later in the curriculum. The idea of defining a concept is at variance with a child's everyday experience where a perceived concept such as "bird" is developed through encounters which begin to focus on salient features. "That is a bird. ... It flies, ... has wings ... and feathers ... and a beak ... and lays eggs". New creatures against these various critieria: "Is a chicken a bird ? ... It has wings, feathers, a beak and lays eggs, but it doesn't fly. OK, some birds don't fly. We will say a chicken is a bird." "Is a bat a bird? It flies and has wings, but it is really a flying mouse, so it is not a bird." Over a period of time the individual builds a complex of interconnected prototypes which help to test whether newly encountered examples are instances of the general concept. (Smith 1988). It therefore comes as no surprise that students are likely to apply similar criteria when faced with concepts in the mathematics class.

We hypothesize that the students develop "prototype examples" of the function concept, such as: a function is like $y=x^{2}$, or a polynomial, or $1 / x$, or a sine function. When asked if a graph is a function, in the absence of an operative definition, the mind attempts to respond by resonating with these mental prototypes. If there is a resonance, the individual experiences the sensation and responds positively. If there is no resonance, the individual experiences confusion, searching the mind to formulate the reason for failure to obtain a mental match. Positive resonances may be in error if they evoke inappropriate properties of prototypes, for instance, that a function is (usually) described by a formula, or that the graph (such as a circle) looks familiar. Negative resonances may also be in error, for instance strange looking graphs may not be considered functions, or a constant cannot be a function because previously encountered prototypes depend on a variable, and so must vary. 


\section{Students' conceptions of a function}

Following ideas of gathering evidence about student conceptions of functions in Vinner (1983) and Barnes (1988), we asked a group of twenty eight students (aged 16/17) to:

Explain in a sentence or so what you think a function is.

If you can give a definition of a function then do so.

They had studied the notion of a function during the previous year previously and had used functions in the calculus but with little emphasis on the technical aspects of domain, range and so on. None gave satisfactory definitions, but all gave explanations, including the following:

- a function is like an equation which has variable inputs, processes the inputted number and gives an output.

- a process that numbers go through, treating them all the same to get an answer.

- an order which plots a curve or straight line on a graph.

- a term which will produce a sequence of numbers, when a random set of numbers is fed into the term.

- a series of calculations to determine a final answer, to which you have submitted a digit.

- a set of instructions that you can put numbers through.

The majority expressed some idea of the process aspect of function - taking some kind of input and carrying out some procedure to produce an output - but no one mentioned that this only applies to a certain domain of inputs, or that it takes a range of values. Many used technical mathematical words, such as term, sequence, series, set, in an everyday sense, intimating a potential difficulties for both students and teachers in transferring mathematical knowledge.

\section{Graphs as functions}

School mathematics is intended to give students experiences of mathematical activities, rather than plumb the formal depths of logical meaning. The formalities may be mentioned, but they are not stressed because they do not appear to be appropriate until the student has a suitable richness of experience. But the collection of activities inadvertently colours the meaning of the function concept with impressions that are different from the mathematical meaning which, in turn, can store up problems for later stages of development.

To investigate students' concept images of the function concept, we showed the same 28 sixthformers plus 109 students starting their first year of university mathematics nine graph sketches and asked:

Which of the following sketches could represent functions? Tick one box in each case. Wherever you have said no, write a little explanation why by the diagram. 

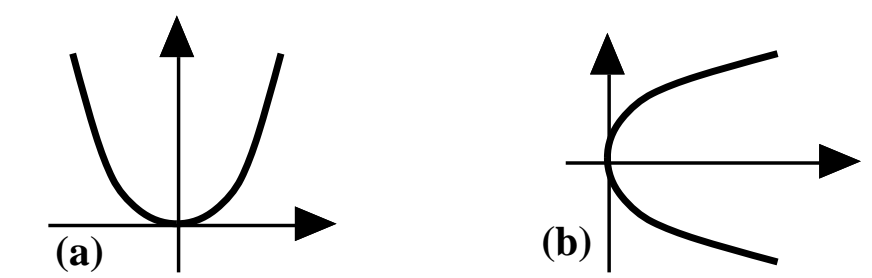

\begin{tabular}{|l|c|c|}
\cline { 2 - 3 } \multicolumn{1}{c|}{} & \% yes & $\%$ no \\
\hline school & $\mathbf{1 0 0}$ & 0 \\
\hline univ. & $\mathbf{9 7}$ & 3 \\
\hline
\end{tabular}

\begin{tabular}{|l|c|c|}
\cline { 2 - 3 } \multicolumn{1}{c|}{} & \% yes & $\%$ no \\
\hline school & 95 & $\mathbf{4}$ \\
\hline univ. & 80 & $\mathbf{2 0}$ \\
\hline
\end{tabular}

These first "starter" questions are in fact ambiguous and we can set little store by them. With the "usual conventions", that the horizontal axis represents the independent variable and the vertical axis the dependent variable, (a) would be adjudged correct and (b) false, (as indicated by printing in heavy type); equally (b) could be true if it represented $x$ as a function of $y$. Only two university students interpreted (b) in the latter manner, one saying "look at it a different way", the other " $f(y)=x$ ". The university students responding negatively to (b) often did so with a comment equivalent to the fact that this "sometimes has two $y$ 's for each $x$ ".

However, they are seen in new light by responses to similar questions using semicircles instead of parabolas:

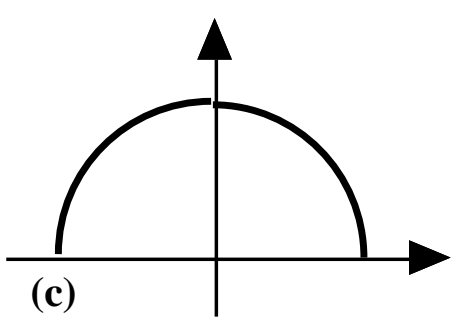

(d)

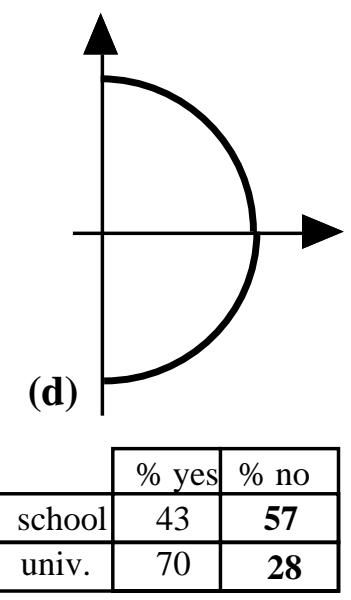

There is a drop to $61 \%$ of school pupils thinking figure (c) is a function whilst it seems that more $(57 \%)$ now correctly respond that figure (d) is not. The drop in belief in figure (c) compared with (a) was accompanied with comments such as:

"if a function the graph would continue, not just stop", "stops dead, values are not limitless", "the lines would have to continue", "this could not apply to any value".

The word "continuous" is here used with the everyday meaning of "continuing without a break". Several of the explanations allude to ideas which suggest that functions should not be unnaturally curtailed. One student extended the graph to "continue" it for more values of $x$. As all functions studied by the students (polynomials, trigonometric functions etc) are defined by a 
formula, this suggests a prototype of a function being "naturally defined everywhere the formula is defined", leading to unease with "artificial" functions such as the top half of a circle.

When faced with a quadrant of a circle, even fewer school pupils considered it a function:

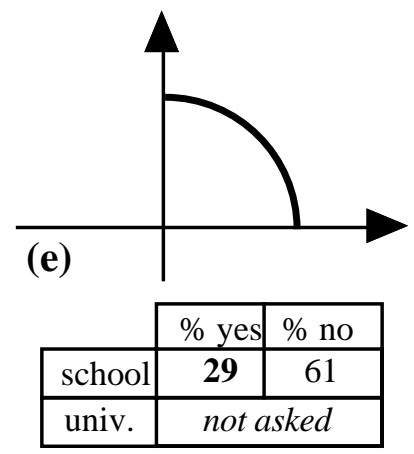

The school pupil's belief in a graph being a function through pictures (a), (c), (e) drops from $100 \%$ to $61 \%$ to $29 \%$ as the graph passes from parabola to semicircle to quadrant, becoming less familiar and restricted to a smaller and smaller domain.

Discussion afterwards revealed that a student who insisted the graph (e) was "not complete" thought of it as part of a circle. For this student a function is a natural totality given by a formula, and it is essential to have it all, not an unnaturally selected part.

Although a quadrant of a circle (which is the graph of a function) is considered not to be a function by most pupils, the situation is reversed with a complete circle. Approximately two thirds of the students in school and university considered the circle in figure (f) to be a function:

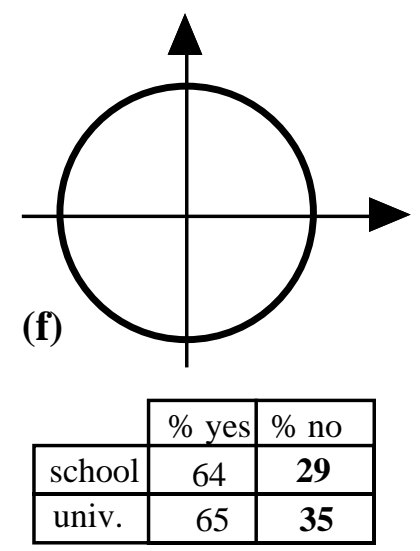

Those thinking it was not a function included two from school saying, "You can't work a function that goes back on itself" or the "equation is $x^{2}+y^{2}=25$ ", implicitly - but not explicitly suggesting that $y$ is not determined uniquely by $x$. Most of the negative responses from the university students alluded to the idea that each value of $x$ might be related to more than one $y$.

The reasons for two thirds of the students thinking that the circle is a function include the possibility that familiarity with the graph evokes the function concept, and that the term "implicit function" (or "many-valued function") is often used (incorrectly) in Britain. 
The final three pictures presented to students - (g), (h) and (i) - presented even more conflict. Both $(\mathrm{g})$ and (h) could satisfy the function definition, but not (i) because there is a part of the graph where one value of $x$ corresponds to more than one value of $y$. However, they look strange, so none of them fit the students' mental collection of prototypes.
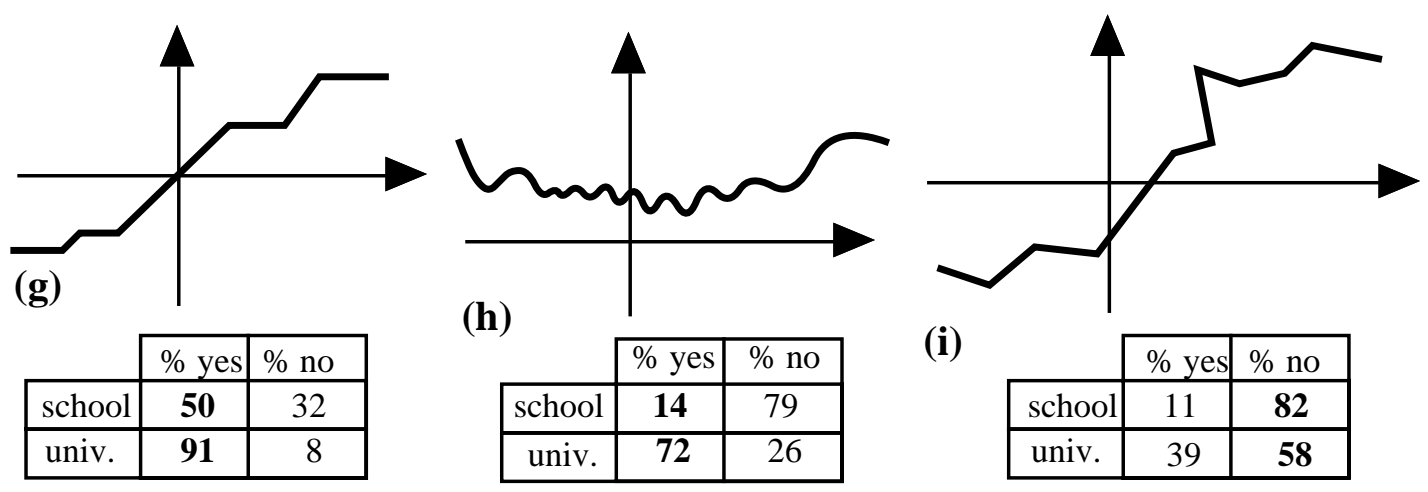

In general the university students appear to cope better with these more general curves. The school pupils greater success with (i) is an illusion, due to its unfamiliarity rather than any formal property of a function:

"graphs are usually smooth, either a straight line or curve, not a combination of the two, nor staggered, when dealing with a function", "these are absurd", "too complicated to be defined as a function", "too irregular", "no regular pattern".

None of these three graphs match the students' mental collection of function prototypes. Their comments support the hypothesis that their prototypes are usually "given by a formula", which tends to have a recognizable shape, have a "smooth" graph, seem "regular" and so on.

Three school pupils do focus on the part of the graph where there are three $y$-values for each $x$ value: "here the curve goes back on itself", ... "there is an irregular peak which could not be created from a function". They are beginning to evoke the restriction that each $x$ should have only one $y$. But they do not apply this test consistently in the earlier examples, and their "definitions" of a function are:

"a mathematical command or identity",

"an equation with a variable factor ... e.g. $\mathrm{f}(x)=x+2$ ",

"the product of a series of numbers which the numbers must undergo".

Not one of the school pupils consistently evokes a coherent function concept. Only eight of the university students ( $7 \%$ of the total) gave a consistent set of replies to all the graphs, with one other consistently allowing $x$ to be a function of $y$ as well as $y$ being a function of $x$.

One graph was given to the university students, but not to those at school (in lieu of graph (e) above): 


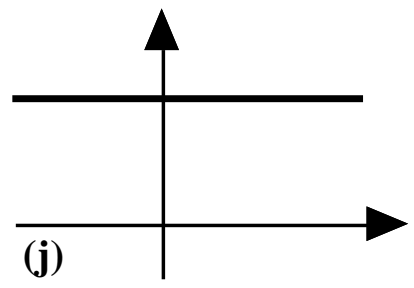

\begin{tabular}{|l|c|c|}
\cline { 2 - 3 } \multicolumn{1}{c|}{} & \% yes & $\%$ no \\
\hline school & \multicolumn{2}{|c|}{ not given } \\
\hline univ. & $\mathbf{5 5}$ & 44 \\
\hline
\end{tabular}

Almost half the students at university think that a constant is not a function, often because $y$ is independent of the value of $x$. Such an interpretation is sometimes given in certain contexts, for instance, asserting that $d y / d x=1 / x$ describes $d y / d x$ as a function of $x$ but not of $y$. Clearly implicit in school mathematics is that the notion of a function has variables, and if a variable is missing, then the expression is not a function of that variable.

\section{Algebraic expressions as functions}

To consider the meaning of a function in terms of formulae (as in Barnes, 1988), we asked the university students to say which of a number of symbolic expressions or procedures could represent $y$ as a function of $x$. Some of these were algebraic equivalents of the pictorial representations mentioned earlier. Thirty eight of the 109 students explicitly mentioned at least once word "many-one" or that for each $x$ there must be one $y$, or equivalent. We include separate columns representing the responses of these 38 "more knowledgeable" students.

\begin{tabular}{|c|c|c|c|c|}
\hline & \multirow{2}{*}{\multicolumn{2}{|c|}{$\begin{array}{l}\text { University } \\
\text { students } \\
(\mathrm{N}=109) \\
\end{array}$}} & & \\
\hline & & & \multicolumn{2}{|c|}{$\begin{array}{c}\text { Subset showing } \\
\text { more knowledge } \\
(\mathrm{N}=38) \\
\end{array}$} \\
\hline & $\%$ yes & $\%$ no & $\%$ yes* & $\%$ no* \\
\hline (1) $y=x^{2}$ & 96 & 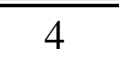 & 95 & 3 \\
\hline (2) $y=4$ & 30 & 69 & 47 & 53 \\
\hline (3) $x^{2}+y^{2}=1$ & 62 & 37 & 40 & 60 \\
\hline (4) $y=3 / x$ & 91 & 9 & 84 & 16 \\
\hline (5) $x y=5$ & 82 & 17 & 82 & 18 \\
\hline (6) $y= \pm \sqrt{4 x-1}$ & 67 & 33 & 34 & 66 \\
\hline (7) $y=\left\{\begin{array}{ccc}0 & \text { if } & x \leq 0 \\
x & \text { if } & 0 \leq x \leq 1 \\
2-x & \text { if } & x>1\end{array}\right.$ & 92 & 7 & 95 & 5 \\
\hline (8) $y=0$ if $x$ is a rational number & 50 & 48 & 42 & 58 \\
\hline (9) $\begin{aligned} y & =0 \text { (if } x \text { is a rational number), } \\
y & =1 \text { (if } x \text { is an irrational number). }\end{aligned}$ & 75 & 22 & 79 & 21 \\
\hline
\end{tabular}


Once again the expression $y=x^{2}$ is almost universally regarded as a function, but the constant $y=4$ is not. As in Barnes (1988), a majority of all students consider the circle $x^{2}+y^{2}=1$ to be a function. Although those showing more technical knowledge perform better, still only $47 \%$ think that $y=4$ is a function whilst $60 \%$ think $x^{2}+y^{2}=1$ is not.

Expressions (4) and (5) show that the majority of students see $y=3 / x, x y=5$ as functions, the major obstacle for the first being that it is not defined for $x=0$, and for the second, not only is it not defined for $x=0$, but the expression is not considered a function until it has been manipulated to get " $y$ as an expression involving $x$ ". The latter is a common function prototype.

Expression (6) shows that the majority of students think that $y= \pm \sqrt{4 x-1}$ is a function. It resonates with the " $y$ equals an expression in $x$ " prototype even though $y$ is not given uniquely. The "more knowledgeable" minority show a marked improvement because they are consciously aware that a function must give (at most) one value of $y$ for each value of $x$.

Expressions (7), (8) and (9) address the problems of defining functions differently on different sub-domains. These do not fit the prototypes familiar to most students. The correct response to (7) is remarkably high given that Vinner 1983 and others have found that function prototypes usually involve only a single formula. No student made such a comment, perhaps becauae in this case each formula on the subdomains is familiar and the function is everywhere defined. The fact that (8) is not everywhere defined definitely caused problems because:

" $y$ is not defined for all $x$ ", or "doesn't state what $y$ is if $x$ is not rational",

Expressions (8) and (9) provoke difficulties because they fail to fit the students' prototypes.

"no real link with $x$, i.e. not actually applying a function to $x$, where the answer would be $y$ ", " $y$ is not in proportion to $x$ ", "no relation between $x$ and $y$ ", "not continuous on the real number line".

\section{Conflicts with constant functions}

Comparing the student responses to the expression $y=4$ and the graph of $y=$ constant, only $28 \%$ reply correctly in the affirmative to both. Table 2 correlates the responses (with the "more knowledgeable" subset percentages in brackets).

\begin{tabular}{|c|c|c|c|}
\hline \multirow{2}{*}{\multicolumn{2}{|c|}{$\begin{array}{l}\text { Is } y=\text { const } \\
\text { a function? }\end{array}$}} & \multicolumn{2}{|c|}{ algebra } \\
\hline & & $\%$ yes & $\%$ no \\
\hline $\begin{array}{l}\mathrm{g} \\
\mathrm{r}\end{array}$ & $\%$ yes & $28(42 *)$ & $29(26 *)$ \\
\hline $\begin{array}{l}\mathrm{p} \\
\mathrm{h}\end{array}$ & $\%$ no & $3\left(5^{*}\right)$ & $41\left(26^{*}\right)$ \\
\hline
\end{tabular}

Table 2 
There is evidence of conflict in a significant number of scripts, as students change their mind when realizing that the algebraic expression clearly does not involve $x$, but the graph seems more likely to be a function. One student who thought initially that $y=4$ was not a function, then wrote it as $y=4 x^{0}$, hence obtaining "a formula involving $x$ ". This may very well be related to the description of the relationship between $x$ and $y$ in terms of variables: that the dependent variable $y$ varies as the independent variable $x$ varies. The expression $y=4$ offends this prototype because $y$ does not vary!

\section{The circle as a function}

Comparing the responses to the graphic and algebraic representations of a circle, we find that $52 \%$ erroneously regard both graph and expression as representing functions, $12 \%$ say "yes" to graph and "no" to expression, $10 \%$ say "no" to graph and "yes" to expression, and only $25 \%$ correctly say "no" to both (table 3 ). The more technical responses increase the percentage correct from $25 \%$ to $47 \%$ - still less than half.

\begin{tabular}{|c|c|c|c|}
\hline \multirow{2}{*}{\multicolumn{2}{|c|}{$\begin{array}{l}\text { Is } y=\text { const } \\
\text { a function? }\end{array}$}} & \multicolumn{2}{|c|}{ algebra } \\
\hline & & $\%$ yes & $\%$ no \\
\hline $\begin{array}{l}\mathrm{g} \\
\mathrm{r}\end{array}$ & $\%$ yes & $28(42 *)$ & $29(26 *)$ \\
\hline $\begin{array}{l}\mathrm{p} \\
\mathrm{h}\end{array}$ & $\%$ no & $3\left(5^{*}\right)$ & $41\left(26^{*}\right)$ \\
\hline
\end{tabular}

Table 3

The position is worse when we consider which students give a correct response to both questions in algebraic and graphic modes:

Only $11 \%$ of all students assert both that $y=$ constant is a function and a circle is not. The percentage only increases to $29 \%$ among the more technical responses.

Thus, even amongst the most able students in the sixth form, the vast majority do not have a coherent concept of function at the end of their A-level studies.

\section{Reflections}

Because the general function concept is difficult to discuss in full generality we take the pragmatic route of de-emphasizing theory and emphasizing practical experience. Attempts to teach the formal theory, as in the New Mathematics of the sixties, have proved unsuccessful. But the other side of the coin - teaching the concept through examples, as in the current curriculum - leads to mental prototypes which give erroneous impressions of the general idea of a function. Even amongst the students who receive some training in the notion of a function, only a small minority respond coherently and consistently. 
We seem to face a formidable, fundamental obstacle:

The learner cannot construct the abstract concept of function without experiencing examples of the function concept in action, and they cannot study examples of the function concept in action without developing prototype examples having built-in limitations that do not apply to the abstract concept.

It is the awareness of this obstacle which should be a major focus of future research to help students cope with the fundamental and necessary mental reorganisation which accompanies the conception of mathematical definitions.

\section{References}

Barnes M 1988: "Understanding the Function Concept: Some Results of Interviews with Secondary and Tertiary Students", Research on Mathematics Education in Australia, 24-33.

Department of Education and Science 1989: Mathematics in the National Curriculum, HMSO, London.

Even R. 1988: Prospective secondary mathematics teachers' knowledge and understanding about mathematical function, unpublished Ph.D. thesis, Michigan State University.

Smith E.E. 1988: "Concepts and thought", in The psychology of human thought, editors Sternberg R.J and Smith E.E., Cambridge University Press, 19-49.

Tall D.O. 1990: "The transition to advanced mathematical thinking: functions, limits, infinity and proof", Handbook on Research in Mathematics Education, N.C.T.M., (to appear).

Vinner S. 1983: "Concept definition, concept image and the notion of function", The International Journal of Mathematical Education in Science and Technology, 14, 293-305. 